\title{
Investigating a hyper-endemic focus of Taenia solium in northern Lao PDR
}

Anna Okello ${ }^{1,24^{*}}$, Amanda Ash ${ }^{3}$, Chattouphone Keokhamphet ${ }^{2}$, Emma Hobbs ${ }^{4}$, Boualam Khamlome ${ }^{5}$, Pierre Dorny ${ }^{6}$, Lian Thomas ${ }^{7,8}$ and John Allen ${ }^{1}$

\begin{abstract}
Background: The Taenia solium cysticercosis-taeniasis complex is a Neglected Tropical Disease of significant public health importance in many impoverished communities worldwide. The parasite is suspected to be endemic in Lao PDR as a result of widespread risk factors including open human defecation, free ranging pigs and weak systems for meat inspection and carcass condemnation. Reported prevalences of human taeniasis throughout the country have ranged from 0-14\%, although few of these have definitively diagnosed T. solium, grossly indistinguishable from Taenia saginata (beef tapeworm) and Taenia asiatica. This short communication details the suspicion of a hyper endemic "hotspot" of T. solium in a remote Tai Dam village in northern Lao PDR.
\end{abstract}

Findings: Initial antibody serosurveillance of four provinces in Lao PDR in 2011 indicated human taeniasis and cysticercosis prevalences of $46.7 \%$ and $66.7 \%$ respectively, in the village of Om Phalong in the north of the country. Subsequent copro-antigen ELISA on 92 human faecal samples from this same village, representing a total $27.9 \%$ of the target community, indicated a taeniasis prevalence of $26.1 \%$ ( $95 \% \mathrm{Cl}=18.2-35.9$ ). Subsequent PCR and sequencing of samples $(n=5)$ all identified as T. solium; the other human tapeworms T. saginata and T. asiatica were not detected in any of the samples genotyped.

Conclusion: This is potentially one of the highest documented prevalences of T. solium taeniasis to date in Lao PDR, if not the Southeast Asia region. This result raises suspicion that other "hotspots" of T. solium hyper endemicity may exist in the region, particularly in communities where the consumption of raw pork is commonplace as a result of cultural practices.

Keywords: Taenia solium, Taeniasis, Cysticercosis, Lao PDR, Neglected tropical diseases, One health

\section{Findings \\ Background}

The human taeniasis/cysticercosis complex, caused by the tapeworm parasite Taenia solium, is listed by the World Health Organisation (WHO) as one of eight Neglected Zoonotic Diseases (NZDs) of significant public health importance, for which a combined 'One Health' approach by the human and animal health sectors is recommended for control in endemic countries [1,2]. Humans can act as both the definitive and accidental dead-end intermediate

\footnotetext{
* Correspondence: anna.okello@ed.ac.uk

${ }^{1}$ CSIRO Animal, Food and Health Sciences, Australian Animal Health Laboratory (AAHL) Regional Programme, 5 Portarlington Road, East Geelong, Victoria, Australia

${ }^{2}$ National Animal Health Laboratory, Department of Livestock and Fisheries, Lao PDR, Vientiane, Lao

Full list of author information is available at the end of the article
}

hosts of the parasite, with a risk of infection from both the adult (taeniasis) and larval stages (cysticercosis), respectively. Poor sanitation, open defecation and free-ranging pig production systems contribute to the ingestion of tapeworm eggs by the human and pig intermediate hosts, leading to the development of larval cysts in both. In humans, a common site of cyst development is the brain, manifested as a condition known as neurocysticercosis (NCC) and deemed the most frequent preventable cause of acquired epilepsy in endemic countries [3,4]. In addition, ingestion of cystic pork through the consumption of raw or undercooked meat propagates parasite survival in at-risk human populations through increasing the population of taeniasis positive carriers. Advocacy and political commitment for the control of T. solium in endemic countries under various Neglected Tropical Disease (NTD) frameworks is growing, culminating with the World Health Assembly's adoption of Resolution 
WHA66.12 on NTDs in May 2013. Prior to this, specific Action Points in the 2012 WHO NTD Roadmap referred to the requirement by 2015 for a "validated strategy for the control and elimination of T. solium", with scaling up of interventions in selected countries by 2020 [5].

The Lao People's Democratic Republic (Lao PDR) is a Southeast Asian country that relies heavily on agricultural production, with up to $70 \%$ of households in the northern part of the country involved in smallholder pig production [6]. The conditions perpetuating T. solium transmission are widespread, including free ranging pig production systems, poor sanitation and lack of meat inspection, particularly at informal markets. A number of surveys on human intestinal helminth infections in different parts of the country over the last 25 years have identified non-specific Taenia species, with prevalences ranging from 0 to $14 \%$ [6]. A more recent study across four northern provinces in Lao PDR found a human cysticercosis prevalence of $2.2 \%(95 \% \mathrm{CI}=1.4-3.0)$ and taeniasis prevalence of $8.4 \%$ (95\% CI $=6.9-9.9)$ [7].

In 2011, a large scale multi-disease serosurvey of pigs and humans was undertaken in Savanakhet and Luang Prabang provinces of Lao PDR, with additional samples from eight villages in Phongsaly and Xayaboury provinces in the far north and west, under collaboration between the Australian Centre for International Agricultural Research (ACIAR) and the International Livestock Research Institute (ILRI) [8]. Over 1000 human serum samples $(n=1012)$ from the four provinces were tested for a range of pig zoonoses, including the use of an Enzymelinked Immunoelectrotransfer Blot strip (EITB, US Centers for Disease Control, Atlanta, Georgia) to determine previous exposure of human participants to T. solium. EITB is reported to be highly specific (100\%) and sensitive (97\%) to the detection of circulating T. solium taeniasis antibodies, with a cysticercosis specificity and sensitivity of $99.4 \%$ and $97 \%$ respectively, provided at least two cysts are in the brain [9]. A number of hotspots were identified amongst the 59 surveyed villages, one of which was the remote Tai Dam village of Om Phalong in Mai District, Phongsaly Province in the north of the country. Abnormally high antibody prevalences of $T$. solium taeniasis (46.67\%, 95\% CI = 22-72) and cysticercosis $(66.67 \%, 95 \%$ $\mathrm{CI}=38-86)$ were detected on the initial EITB survey in this village, compared to an overall prevalence of $2.9 \%$ (taeniasis) and $4.7 \%$ (cysticercosis) in the wider survey (unpublished data). The large confidence intervals in this case reflect the relatively small sample population $(n=16)$ determined under the proportional to size sampling frame for the wider provincial-level study. The requirement for follow up work in Om Phalong village to confirm the suspected hotspot was acknowledged by researchers, who returned in January 2013 to investigate further; the results of which are discussed below.

\section{Methods}

The village of Om Phalong is situated in a remote mountainous region of Mai District in Phongsaly province, close to the Vietnamese border in northern Lao PDR. Its 330 inhabitants practice subsistence and swidden agriculture, with the village largely inaccessible for five to six months of the year during the wet season. All village members are from the Tai Dam or "Black Tai" ethnic group, an animistic culture of which the ritual slaughtering and subsequent raw consumption of pigs and buffalo occurs throughout the year around major ancestral ceremonies [10].

Sample collection took part over a two week period during the 2013 dry season, when village access was maximised and the rice harvest complete. All village members over 10 years old were invited to participate in the study, via the completion of a questionnaire (accompanied by the parent or guardian in the case of the volunteer being less than 16 years of age) and the submission of a faecal sample. Originally, serum samples were also requested to gauge the level of active cysticercosis in the village, however overlying cultural sensitivities of the Tai Dam around withdrawal of blood from the body meant only thirteen venous blood samples were collected during this time. After informed consent through a signature or thumb print, participants were given a labelled plastic bag for a faecal sample to be returned within 24 hours. Participants were identified so they could be traced back for treatment if found to be positive. Ethical approval was obtained from the Lao PDR Ministry of Health National Ethics Committee for Health Research (NECHR) [approval number 013/NECHR].

At the conclusion of every day, collected faecal samples were divided into three $10 \mathrm{ml}$ falcon tubes containing $10 \%$ formalin, 5\% formal saline and 70\% ethyl alcohol respectively, allowing for analysis by microscopy, copro-antigen ELISA and PCR. Upon return to Vientiane, samples in 5\% formal saline were processed in-country by a coproantigen ELISA (ITM, Belgium) to determine excretorysecretory proteins from adult Taenia species, as discussed in Allan and Craig [11]. In addition to identifying Taenia sp. by copro-antigen ELISA, all 92 faecal samples underwent microscopy analysis for Taenia sp. ova and co-existing Soil Transmitted Helminths (STH), expanding the parasitological baseline in order to demonstrate any added value to a future T. solium control intervention. All copro-antigen ELISA and microscopy positive samples were subsequently submitted for molecular characterisation at Murdoch University in Western Australia. Additionally, the thirteen collected blood samples were tested for human cysticercosis using the Advanced Practical Diagnostics Cysticercosis Antigen (Ag) ELISA (apDia, Belgium, Reference 650501), reporting a diagnostic sensitivity of 100 percent provided three or more viable cysts are present [12]. 


\section{Results and discussion}

A total of 92 participants, representing $27.9 \%$ of the total village population, were included in the final dataset, having supplied a faecal sample, participated in the questionnaire, and complied with ethical guidelines of participation. Initial microscopy analysis identified Taenia spp. ova in 8 samples. All 92 samples then underwent analysis by copro-antigen ELISA, with the 8 microscopy positive samples and a further 16 samples ( 24 in total) testing positive, indicating a human taeniasis prevalence of $26.1 \%$ ( $95 \% \mathrm{CI}=18.2-35.9$ ). The 24 copro-antigen positive samples were then submitted for PCR at the 12S rRNA locus as per Traschel et al. [13] at Murdoch University in Perth, Western Australia. A generic taeniid PCR was used due to a need to detect Taenia sp. from more than one host (not reported here). However, only those eight samples for which Taenia ova were identified on microscopy amplified successfully, due to the requirement for Taenia DNA to be present in order for amplification to occur [14]. Of the eight samples positive by PCR five sequenced successfully, with three failing the sequencing process. All five however, identified 100\% with published genetic sequences for T. solium [NCBI Accession numbers AB086256 and L49444].

Out of the thirteen submitted blood samples, four were found to be positive, indicating a presence of circulating antigens to T. solium cysticerci of 30\% (95\% CI 9-61). Despite the large confidence interval and potential sampling bias in these thirteen samples, subsequent social research revealed that of the four cysticercosis positive patients, only one wanted to have their blood taken due to feeling "ill", with the other three submitting blood samples because they "wanted to participate in the village activity" (Key Informant Interviews, 2013). A broader investigation under the aforementioned anthropological study revealed complaints of headaches, dizziness and subcutaneous nodules to be relatively widespread in the village (unpublished data). Despite very few reports of seizures or epilepsy from both quantitative and qualitative interview techniques, further longitudinal investigation is required, given the potentially sensitive subject matter for many ethnic groups in the region [15].

The fact that $T$. saginata and/or $T$. asiatica were not detected in any of the genotyped samples, coupled with the abnormally high EITB results on the initial 2011 survey and positive human serum-antigen ELISAs, leads us to believe that $T$. solium is indeed the predominant tapeworm within the copro-antigen positive samples. Moreover, the range of complementary diagnostic tests used in this study strongly suggests T. solium exists within the human population of Om Phalong at significantly higher levels than indicated in previously published studies from Lao PDR [6,7]. We consider this discovery may be the tip of the iceberg of potential hyper-endemic hotspots of $T$. solium in the country, particularly for certain ethnic groups such as the Tai Dam, for which raw pork is the preferred method of consumption during sacrificial slaughter. In terms of control in this particular village, a combined human and porcine biomedical intervention is currently underway in an attempt to significantly lower the current taeniasis burden, thus impacting on further incidence of human cysticercosis infections in the short term. In order to address the underlying sanitation issues and develop robust strategies for longer term control, social research methodologies will continue to be applied in parallel to the epidemiological investigation, in order to explore the various socioeconomic and sociocultural drivers of T. solium transmission in this particular ethnic group.

\section{Conclusion}

It is envisaged that the information gained through the use of combination diagnostic and methodological approaches implemented here will assist the development of longer term, locally acceptable strategies for control, which could eventually be rolled out into other similarly affected communities in the region. Given the suspected high clustering of T. solium infection in Lao PDR, possibly attributed to cultural norms of raw pork eating by specific ethnic groups, the authors recommend further epidemiological and socio-cultural research approaches to refine the combination methodologies applied in this investigative study.

\section{Abbreviations \\ AAHL: Australian Animal Health Laboratory of the CSIRO; ACIAR: Australian Centre for International Agricultural Research; CSIRO: Commonwealth Scientific and Industrial Research Organisation; EITB: Enzyme-linked Immunoelectrotransfer Blot strip; ELISA: Enzyme-linked immunosorbent assay; ILRI: International Livestock Research Institute; Lao PDR: Lao People's Democratic Republic; NTD: Neglected tropical disease; NZD: Neglected Zoonotic Disease; PCR: Polymerase chain reaction; WHA: World Health Assembly; WHO: World Health Organisation.}

\section{Competing interests}

The authors declare that they have no competing interests.

\section{Authors' contributions}

$\mathrm{AO}, \mathrm{AA}, \mathrm{BK}$ and $\mathrm{JA}$ designed the study. $\mathrm{AO}, \mathrm{AA}, \mathrm{CK}, \mathrm{BK}$ and $\mathrm{EH}$ undertook sample collection. AA, CK and LT conducted the laboratory analysis and $A O$ drafted the manuscript, with subsequent input from AA, LT, EH, PD and JA. All authors read and approved the final manuscript.

\section{Acknowledgements}

The project would like to thank the Lao PDR Ministry of Health for their support and assistance in undertaking the field work; in particular the staff from the Mai District and Phongsaly Provincial Health Offices. This research was funded by the Australian Centre for International Agricultural Research (ACIAR) Animal Health Programme, grant AH2009/001 and AH/2009/019.

\section{Author details}

${ }^{1}$ CSIRO Animal, Food and Health Sciences, Australian Animal Health Laboratory (AAHL) Regional Programme, 5 Portarlington Road, East Geelong, Victoria, Australia. ${ }^{2}$ National Animal Health Laboratory, Department of Livestock and Fisheries, Lao PDR, Vientiane, Lao. ${ }^{3}$ School of Veterinary and Life Sciences, Murdoch University, Perth, Australia. ${ }^{4}$ School of Biomedical Sciences, College of Medicine and Veterinary Medicine, University of Edinburgh, Chancellors Building 49 Little France Crescent, Edinburgh EH16 4SB, Scotland. ${ }^{5}$ Department of Hygiene and Prevention, Ministry of Health, 
Lao PDR, Vientiane, Lao. ${ }^{6}$ Veterinary Helminthology Unit, Department of Biomedical Sciences, Institute of Tropical Medicine, Nationalestraat 155, 2000 Antwerp, Belgium. ${ }^{7}$ International Livestock Research Institute, PO Box 30709, Nairobi 00100, Kenya. ${ }^{8}$ Centre for Immunity, Infection and Evolution

University of Edinburgh, Kings Buildings, West Mains Rd, Edinburgh, Scotland EH9 3YJ.

Received: 17 December 2013 Accepted: 12 March 2014

Published: 28 March 2014

\section{References}

1. World Health Organisation: Integrated Control of Neglected Zoonoses in Africa: Adapting the "One Health" concept 2009. Report of a joint WHO/ EU/ILRI/DBL/FAO/OIE/AU Meeting, 13-15 November 2007. Nairobi, Kenya: ILRI Headquarters.

2. Molyneux D, Hallaj Z, Keusch GT, McManus DP, Ngowi H, Cleaveland S, Ramos-Jimenez P, Gotuzzo E, Kar K, Sanchez A, Garba A, Carabin H, Bassili A, Chaignat CL, Meslin F-X, Abushama H, Willingham AL, Kioy D: Zoonoses and marginalised infectious diseases of poverty: Where do we stand? Parasit Vectors 2011, 4(106). doi: 10.1186/1756-3305-4-106.

3. Ndimubanzi PC, Carabin H, Budke CM, Nguyen $\mathrm{H}$, Qian $\mathrm{Y}-\mathrm{J}$, Rainwater $\mathrm{E}$, Dickey M, Reynolds S, Stoner JA: A Systematic Review of the Frequency of Neurocysticercosis with a Focus on People with Epilepsy. PLoS Negl Trop Dis 2010, 4(11):e870. doi: 10.1371/journal.pntd.0000870.

4. Del Brutto OH, Rajshekar V, White AC Jr, Tsang VC, Nash TE, Takayanagui OM, Schantz PM, Evans CAW, Flisser A, Correa D, Botero D, Allan JC, Sarti E, Gonzalez AE, Gilman RH, García HH: Proposed diagnostic criteria for neurocysticercosis. Neurol 2001, 57:177-183.

5. World Health Organisation: Accelerating work to overcome the global impact of neglected tropical diseases - A roadmap for implementation. Uniting to Combat Neglected Tropical Diseases, Ending the Neglect and Reaching 2020 Goals, 2012, London.

6. Conlan J, Khounsy S, Inthavong P, Fenwick S, Blacksell S, Thompson RCA: A review of taeniasis and cysticercosis in the Lao People's Democratic Republic. Parasitol Int 2008, 57:252-255.

7. Conlan J, Vongxay K, Khamlome B, Dorny P, Sripa B, Elliot A, Blacksell SB, Fenwick S, Thompson RCA: A Cross-Sectional Study of Taenia solium in a Multiple Taeniid-Endemic Region Reveals Competition May be Protective. Am J Trop Med Hyg 2012, 87(2):281-291.

8. Inthavong P, Khamlone B, Phimphachanhvongsod V, Blaszak K, Allen J, Durr P Gilbert J, Donnelly B: A Participatory Ecohealth Study of Smallholder Pig Systems in Upland and Lowland Lao PDR. Oral presentation Fourth Biennial Conference of the International Association for Ecology and Health 2012. [http://ecohealth-live.net/wp-content/uploads/2012/10/Abstractsbook_EcoHealth2012_October-13.pdf]

9. Deckers N, Dorny P: Immunodiagnosis of Taenia solium taeniosis/ cysticercosis. Trends Parasitol 2010, 26:137-144.

10. Chazée L: The Peoples of Laos: Rural and Ethnic Diversities. White Lotus Bangkok; 2002. ISBN 974-8434-86-9.

11. Allan JC, Craig PS: Coproantigens in taeniasis and echinococcosis. Parasitol Int 2006, 55(Supplement 1):S75-S80.

12. Advanced Practical Diagnostics (apDia). [http://www.apdiagroup.com/ sites/default/files/files/apDia\%20\%20dedicated\%20newsletter\%201\%20-\% 20cysticercosis.pdf]

13. Trachsel D, Deplazes $P$, Mathis A: Identification of taeniid eggs in the faeces from carnivores based on multiplex PCR using targets in mitochondrial DNA. Parasitol 2007, 2007(134):911-920.

14. Praet N, Verweij J, Mwape K, Phiri I, Muma J, Muma JB, Zulu G, van Lieshout L, Rodriguez-Hidalgo R, Benitez-Ortiz W, Dorny P, Gabriël S: Bayesian modelling to estimate the test characteristics of coprology, coproantigen ELISA and a novel real-time PCR for the diagnosis of taeniasis. Trop Med Int Health 2013:1-7. doi: 10.1111/tmi.12089.

15. Fadiman A: The Spirit Catches you and you Fall Down: a Hmong Child, her American Doctors, and the Collision of Two Cultures. New York: Noonday; 1998.

doi:10.1186/1756-3305-7-134

Cite this article as: Okello et al:: Investigating a hyper-endemic focus of Taenia solium in northern Lao PDR. Parasites \& Vectors 2014 7:134.

\section{Submit your next manuscript to BioMed Central and take full advantage of:}

- Convenient online submission

- Thorough peer review

- No space constraints or color figure charges

- Immediate publication on acceptance

- Inclusion in PubMed, CAS, Scopus and Google Scholar

- Research which is freely available for redistribution

Submit your manuscript at www.biomedcentral.com/submit
() Biomed Central 